\title{
VISITA DOMICILIAR A IDOSOS: CARACTERÍSTICAS E FATORES ASSOCIADOS
}

\section{HOME VISIT TO ELDERLY: FEATURES AND ASSOCIATED FACTORS}

\section{VISITA DOMICILIAR A LOS ANCIANOS: CARACTERISTICAS Y FACTORES ASOCIADOS}

\author{
Gerson Souza Santos ${ }^{1}$, Isabel Cristina Kowal Olm Cunha ${ }^{2}$
}

\section{RESUMO}

Objetivo: analisar os fatores associados à visita domiciliar na população idosa e suas características segundo os preceitos da Estratégia Saúde da Família. Método: estudo transversal de base populacional com amostra representativa de 340 indivíduos com 60 anos ou mais residentes na zona urbana de São Paulo, SP. Resultados: a única variável que apresentou efeito estatisticamente significativo na visita domiciliar foi "passar em consulta médica na UBS" ( $p$-valor = 0,0022). O odds ratio estimado para essa variável foi de 2,369 , com intervalo de $95 \%$ de confiança. Conclusão: os resultados do presente estudo mostraram que os idosos avaliados eram mulheres na faixa etária de 60 a 69 anos, com baixa escolaridade, renda familiar insuficiente, vivendo sem cônjuge, dependentes do Sistema único de Saúde, havendo presença de doenças crônicas com destaque para hipertensão arterial e incapacidade para desempenho de atividades instrumentais da vida diária.

Descritores: Atenção primária à saúde; Visita domiciliar; Envelhecimento da população.

\section{ABSTRACT}

Objective: to analyze the factors associated with home visits in the elderly population and their characteristics, in accordance with the principles of the Family Health Strategy. Method: A cross-sectional population-based study with a representative sample of 340 individuals aged 60 years or older residing in the urban area of São Paulo, SP. Results: The only variable that presented a statistically significant effect on the home visit was a medical visit at the UBS ( $p$-value $=0.0022$ ). The estimated odds ratio for this variable was 2,369, with a $95 \%$ confidence interval. Conclusion: the results of the present study showed that the evaluated elderly were the women in the age group of 60 to 69 years, low schooling, insufficient family income, living without spouse, dependents of the Single Health System, with the presence of chronic diseases with a prominence for hypertension arterial, inability to perform instrumental activities of daily living.

Descriptors: Primary health care, Home visit, population aging.

\section{RESUMEN}

Objetivo: analizar los factores asociados a la visita domiciliaria en la población anciana y sus características, según los preceptos de la Estrategia Salud de la Familia. Método: Estudio transversal de base poblacional con muestra representativa de 340 individuos con 60 años o más residentes en la zona urbana de São Paulo, SP. Resultados: La única variable que presentó efecto estadísticamente significativo en la visita domiciliaria fue pasar en consulta médica en la UBS ( $p$-valor $=0,0022$ ). El odds ratio estimado para esta variable fue de 2,369 , con un intervalo de confianza del $95 \%$. Conclusión: los resultados del presente estudio mostraron que los ancianos evaluados eran las mujeres en el grupo de edad entre 60 y 69 años, baja escolaridad, ingreso familiar insuficiente, viviendo sin cónyuge, dependientes del Sistema Único de Salud, presencia de enfermedades crónicas con destaque para hipertensión, incapacidad para el desempeño de actividades instrumentales de la vida diaria.

Descriptores: Atención primaria a la salud, Visita domiciliar, Envejecimiento de la población.

${ }^{1}$ Graduado em Enfermagem. Doutor em Ciências da Saúde pela Universidade Federal de São Paulo. ${ }^{2}$ Graduada em Enfermagem. Professora Titular do Departamento de Enfermagem da Universidade Federal de São Paulo.

Como citar este artigo:

Santos GS, Cunha ICKO. Visita domiciliar a idosos: características e fatores associados. Revista de Enfermagem do Centro-Oeste Mineiro. 2017;7:e1271. https://doi.org/10.19175/recom.v7i0.1271 


\section{INTRODUÇÃO}

O processo de envelhecimento humano tem sido tema de discussão em quase todos os países do mundo e, no Brasil, toma proporções alarmantes, visto que a estimativa de vida da população tem aumentado significativamente. Isso se deve à melhoria das condições de vida, de saneamento básico, de trabalho, de educação, bem como das condições tecnológicas que possibilitaram que se vivesse mais e com melhor qualidade $^{(1)}$.

As pessoas idosas apresentam características especiais quanto à natureza de seus agravos, ao modo de adoecimento e ao uso dos serviços de saúde, o que exige um amplo redimensionamento das práticas de saúde para fazer em face das novas demandas impostas pela crescente população de longevos. Torna-se prioridade a implementação de serviços e programas inovadores, curto-efetivos, e que incorporem novos paradigmas da atenção à saúde com foco na capacidade funcional muito mais do que na doença ${ }^{(2)}$.

Embora envelhecer não signifique diretamente adoecer e estar dependente, sem dúvida indica uma maior fragilidade e vulnerabilidade que aumenta conforme a idade cronológica dos indivíduos é mais avançada, aliada ao contexto social e ambiental em que o idoso se insere. $\mathrm{Na}$ maior parte dos casos, a responsabilidade de cuidados ao idoso fragilizado é assumida pela família, que nem sempre está preparada para tal condição. Esse cuidado se concretiza nas ações cotidianas da vida diária e envolve apoio funcional, social, econômico, material e afetivo ${ }^{(3)}$.

O desafio do envelhecimento perante a assistência à saúde ocorre em função da estreita relação entre utilização de serviços de saúde e idade. Por isso, os gastos com saúde crescem em uma população que envelhece. Para estimar o impacto no Brasil, utilizam-se os cálculos do Instituto de Estudos de Saúde Suplementar (IESS) que medem regularmente as despesas anuais e sua variação para um conjunto de 1,1 milhão de beneficiários de planos individuais de saúde, distribuídos nas dez faixas etárias da regulação ${ }^{(4)}$.

Uma das estratégias adotada pelos serviços para desonerar o Estado e modificar o modo tradicional de produção em saúde é a inclusão da visita domiciliar no rol de modalidades de atendimento, a qual se caracteriza pela visita da equipe de saúde ao domicílio do usuário com o objetivo de avaliar suas necessidades e as de sua família, considerando a disponibilidade do serviço e constando de plano assistencial e orientações. A visita domiciliar pressupõe uma ação complexa, exigindo técnica e periodicidade da equipe de saúde, de acordo com as necessidades evidenciadas ${ }^{(5)}$.

Essa modalidade tem resultado em maior conforto e segurança ao idoso e sua família, bem como proporcionando cuidado humanizado e qualidade de vida, se comparada ao atendimento institucional. Mas a concreção dessa perspectiva requer um diagnóstico situacional favorecedor de informações quantitativas e qualitativas que contribuam efetivamente para o conhecimento da realidade de saúde da população idosa e da sua dinâmica domiciliar e familiar. As orientações propiciadas por essas informações permitem a tomada de decisões, de forma que o planejamento do serviço de saúde esteja organizado pelo princípio da equidade, priorizando aqueles que mais necessitem de cuidados $^{(6)}$.

No Brasil, nos anos noventa, a concepção de Atenção Primária à Saúde (APS) também foi renovada. Com a regulamentação do Sistema Único de Saúde baseada na universalidade, equidade, integralidade e nas diretrizes organizacionais de descentralização e participação social, para diferenciar-se da concepção seletiva de APS, passou-se a usar o termo atenção básica em saúde, definida como ações individuais e coletivas situadas no primeiro nível, voltadas à promoção da saúde, prevenção de agravos, tratamento e reabilitação ${ }^{(7)}$.

A Estratégia Saúde da Família (ESF) compreende um modelo de atenção à saúde no contexto da Atenção Básica em Saúde, que se estrutura na perspectiva do trabalho de equipes multiprofissionais em um território adstrito desenvolvendo ações a partir do conhecimento da realidade local e das necessidades da população deste território ${ }^{(7)}$.

O objetivo do presente estudo foi analisar os fatores associados à visita domiciliar na população idosa e suas características, segundo os preceitos da Estratégia Saúde da Família, a qual se constitui uma estratégia de reorientação do modelo assistencial. 


\section{MÉTODOS}

Estudo transversal de base populacional, realizado de janeiro a março de 2012. A amostra foi selecionada por conveniência e constituída por 340 indivíduos com 60 anos ou mais, residentes na área de abrangência da Unidade Básica de Saúde Santa Catarina, localizada município de São Paulo.

Foram utilizados questionários estruturados com questões pré-codificadas, após realização de um estudo-piloto. Em caso de incapacidade parcial, as respostas foram dadas por um familiar responsável ou pelo cuidador principal.

A variável dependente 'visita domiciliar' foi definida como uma atividade externa à unidade de saúde caracterizada por utilizar tecnologia leve, permitindo o cuidado à saúde de forma mais humana, acolhedora, estabelecendo laços de confiança entre os profissionais e os usuários, a família e a comunidade. A questão aplicada foi: 'o(a) senhor(a) costuma receber visita da equipe de saúde em sua casa?' (sim/não).

As variáveis demográficas e socioeconômicas estudadas foram: sexo, idade (60 a 69 anos; 70 a 79 anos; 80 anos ou mais); situação conjugal (casado; solteiro; divorciado; ou viúvo); cor da pele (branca; negra; parda); sabe ler/escrever (sim; não); renda per capita (em salários mínimos: até 1 ; de 2 a 3 ; de 4 a 6); plano privado de saúde (sim; não); em caso de doença procura (farmácia; hospital; unidade de saúde); passa em consulta médica na UBS (sim; não); hospitalização no último ano (sim; não). Entre os indicadores de morbidade, foram usadas as variáveis: diagnóstico médico de hipertensão (sim; não); diabetes (sim; não); dislipidemia (sim; não); lombalgia (sim; não); depressão (sim; não); insônia (sim; não). Para estabelecer incapacidade funcional foram utilizadas escalas de avaliação funcional do idoso ${ }^{(8-9)}$.

Para responder ao objetivo do estudo foi utilizado um modelo de Regressão Logística em que a variável dependente foi a visita domiciliar (variável binária, do tipo 'sim' ou 'não') e as variáveis independentes foram as 18 informações acima citadas. Todos os testes de hipóteses desenvolvidos consideraram uma significância de $5 \%$, isto é, a hipótese nula foi rejeitada quando $p$-valor foi menor ou igual a 0,05. A técnica de regressão logística é utilizada quando há o interesse em modelar uma variável dependente binária em função de um conjunto de variáveis independentes. O modelo geral, para o caso de apenas uma variável independente, pode ser escrito da seguinte forma:

$$
\pi(x)=\frac{e^{\beta_{0}+\beta_{1} X}}{1+e^{\beta_{0}+\beta_{1} X}}
$$

Sendo $\pi(x)$ a probabilidade de ocorrência do evento de interesse e, portanto, recebe valores entre 0 e $1, X$ é a variável independente a partir da qual buscamos prever a ocorrência do evento de interesse, $\beta_{0}$ é uma constante que representa a média geral, denominada intercepto, e $\beta_{1}$ é uma constante que, de forma exponencial, influencia a probabilidade de sucesso de acordo com o valor da variável independente $X$.

Trata-se de parte dos resultados extraídos da tese de Doutorado intitulada: Atendimento ao idoso na Atenção Básica e as competências do enfermeiro, defendida em 28/08/2014.

O projeto foi aprovado pelo comitê de Ética em Pesquisa da Universidade Federal de São Paulo (Parecer 2012/11) e da Secretaria Municipal de Saúde do município de São Paulo (Parecer 378/11). Os princípios éticos foram assegurados, recorrendo-se ao Termo de Consentimento Livre e Esclarecido.

\section{RESULTADOS E DISCUSSÃO}

A maioria dos idosos era do sexo feminino, 211 (62\%). A idade variou de 60 a 85 anos, média de 69 anos com desvio padrão de 7,25. Houve predominância para cor da pele parda 143 (42\%). Menos da metade era de viúvos, 115 (34\%). A baixa escolaridade foi frequente e a amostra foi constituída predominantemente por ensino fundamental incompleto. Maior proporção de idosos possuía renda familiar de um a três salários mínimos, 264 (78\%). A maioria não possuía plano privado de saúde, 280 (82\%). Em caso de doença, 154 (46\%) procuravam os hospitais de referência. Hipertensão arterial foi a principal morbidade referida, seguida de diabetes. A maioria passava em consulta médica na Unidade Básica de Saúde, 219 (64\%). Incapacidade para desenvolver atividades instrumentais da vida diária esteve presente em 295 (87\%). Menos da metade 90 (27\%) apresentava incapacidade para realizar atividades básicas da vida diária (Tabela 1).

A única variável que apresentou efeito estatisticamente significativo na visita domiciliar foi a variável 'passar em consulta médica na UBS' ( $p$-valor $=0,0022)$. O odds ratio estimado para essa variável foi de 2,369, com intervalo de $95 \%$ de confiança entre 1,364 e 4,115. Dessa forma, 
podemos concluir que passar em consulta médica na UBS aumenta em 2,369 vezes a chance de receber visita domiciliar, sendo que esse valor pode variar, com $95 \%$ de confiança, entre 1,364 e
4,115. Nenhuma outra variável apresentou efeito estatisticamente significativo na visita domiciliar, uma vez que $p$-valor foi maior que 0,05 em todos os demais casos (Tabela 2).

Tabela 1 - Características socioeconômicas e demográficas de pessoas com 60 anos ou mais, cadastradas em uma Unidade Básica de Saúde - São Paulo, São Paulo (2013).

\begin{tabular}{|c|c|c|}
\hline Variáveis & $\mathrm{n}$ & $\%$ \\
\hline \multicolumn{3}{|l|}{ Sexo } \\
\hline Feminino & 211 & 62,0 \\
\hline Masculino & 129 & 38,0 \\
\hline \multicolumn{3}{|l|}{ Idade (anos) } \\
\hline 60 a 69 & 210 & 61,7 \\
\hline 70 a 79 & 90 & 26,4 \\
\hline 80 ou mais & 40 & 11,9 \\
\hline \multicolumn{3}{|l|}{ Cor da pele } \\
\hline Parda & 143 & 42,0 \\
\hline Branca & 108 & 32,0 \\
\hline Negra & 89 & 26,0 \\
\hline \multicolumn{3}{|c|}{ Situação conjugal } \\
\hline Viúvo & 115 & 34,0 \\
\hline Casado & 103 & 30,0 \\
\hline Solteiro & 83 & 24,0 \\
\hline Divorciado & 39 & 11,0 \\
\hline \multicolumn{3}{|c|}{ Sabe ler e escrever } \\
\hline Sim & 177 & 52,0 \\
\hline Não & 163 & 48,0 \\
\hline \multicolumn{3}{|c|}{ Renda per capita (salários mínimos) } \\
\hline 1 a 3 & 264 & 78,0 \\
\hline 4 а 6 & 76 & 22,0 \\
\hline \multicolumn{3}{|c|}{ Plano privado de saúde } \\
\hline Não & 280 & 82,0 \\
\hline $\operatorname{sim}$ & 60 & 18,0 \\
\hline \multicolumn{3}{|l|}{ Morbidades } \\
\hline \multicolumn{3}{|c|}{ Hipertensão arterial } \\
\hline Sim & 254 & 75,0 \\
\hline Não & 86 & 25,0 \\
\hline \multicolumn{3}{|l|}{ Diabetes } \\
\hline Sim & 132 & 39,0 \\
\hline Não & 208 & 61,0 \\
\hline \multicolumn{3}{|l|}{ Dislipidemia } \\
\hline Sim & 89 & 26,0 \\
\hline Não & 251 & 74,0 \\
\hline \multicolumn{3}{|l|}{ Lombalgia } \\
\hline Sim & 73 & 21,0 \\
\hline Não & 267 & 79,0 \\
\hline \multicolumn{3}{|l|}{ Depressão } \\
\hline $\operatorname{sim}$ & 37 & 11,0 \\
\hline Não & 303 & 89,0 \\
\hline \multicolumn{3}{|l|}{ Insônia } \\
\hline Sim & 54 & 16,0 \\
\hline Não & 286 & 84,0 \\
\hline \multicolumn{3}{|c|}{ Consulta médica } \\
\hline $\operatorname{Sim}$ & 219 & 64,0 \\
\hline Não & 121 & 36,0 \\
\hline \multicolumn{3}{|c|}{ Hospitalização } \\
\hline Não & 220 & 65,0 \\
\hline Sim & 120 & 35,0 \\
\hline \multicolumn{3}{|c|}{ Incapacidade AIVD } \\
\hline $\operatorname{Sim}$ & 295 & 87,0 \\
\hline Não & 45 & 13,0 \\
\hline \multicolumn{3}{|c|}{ Incapacidade ABVD } \\
\hline Não & 249 & 73,0 \\
\hline $\operatorname{sim}$ & 90 & 27,0 \\
\hline Visita domic & & \\
\hline $\operatorname{Sim}$ & 231 & 68,0 \\
\hline Não & 109 & 32,0 \\
\hline
\end{tabular}

Fonte: pesquisa realizada pelos autores com idosos no período de janeiro a março de 2013 . 
Tabela 2 - Regressão logística para visita domiciliar, seguida do cálculo de odds ratio e o respectivo intervalo de confiança - São Paulo, São Paulo (2013).

\begin{tabular}{|c|c|c|c|c|c|c|}
\hline Variável & GL & Wald & p-value & $\begin{array}{l}\text { Odds } \\
\text { Ratio }\end{array}$ & $\begin{array}{l}\text { IC 95\% } \\
\text { Odds Ratio } \\
\text { (inferior) }\end{array}$ & $\begin{array}{l}\text { IC 95\% } \\
\text { Odds Ratio } \\
\text { (superior) }\end{array}$ \\
\hline Faixa etária & 2 & 3.8429 & 0.1464 & - & - & - \\
\hline Sexo & 1 & 0.7899 & 0.3741 & - & - & - \\
\hline Cor da pele & 2 & 0.2068 & 0.9018 & - & - & - \\
\hline Situação conjugal & 3 & 2.1592 & 0.5400 & - & - & - \\
\hline Sabe ler e escrever & 1 & 0.8994 & 0.3429 & - & - & - \\
\hline Renda familiar & 2 & 0.8016 & 0.6698 & - & - & - \\
\hline Plano privado de saúde & 1 & 3.5410 & 0.0599 & - & - & - \\
\hline Em caso de doença procura & 2 & 0.2560 & 0.8798 & - & - & - \\
\hline Hipertensão & 1 & 0.5841 & 0.4447 & - & - & - \\
\hline Diabetes & 1 & 1.7325 & 0.1881 & - & - & - \\
\hline Dislipidemia & 1 & 0.1060 & 0.7447 & - & - & - \\
\hline Lombalgia & 1 & 0.5398 & 0.4625 & - & - & - \\
\hline Depressão & 1 & 0.2643 & 0.6072 & - & - & - \\
\hline Insônia & 1 & 0.2985 & 0.5848 & - & - & - \\
\hline Passa em consulta médica na UBS & 1 & 9.3708 & 0.0022 & 2.369 & 1.364 & 4.115 \\
\hline Hospitalização & 1 & 1.0359 & 0.3088 & - & - & - \\
\hline Incapacidade AIVD & 1 & 0.3672 & 0.5445 & - & - & - \\
\hline Incapacidade ABVD & 1 & 2.5837 & 0.1080 & - & - & \\
\hline
\end{tabular}

Fonte: pesquisa realizada pelos autores com idoso no período de janeiro a março de 2013.

Observou-se neste estudo alto percentual de mulheres idosas classificadas como idosas jovens, na faixa etária de 60 a 69 anos. Estudos recentes apontam que as mulheres constituem a maioria da população idosa em todas as regiões do mundo e as estimativas são de que as mulheres vivam, em média, de cinco a sete anos a mais que os homens ${ }^{(10)}$.

A escolaridade dos idosos deste estudo foi baixa, constituindo-se uma condição social desfavorável para eles já que tem influência no acesso aos serviços de saúde, em oportunidades de participação social e na compreensão de seu tratamento e do seu autocuidado, entre outros. A situação de analfabetismo pode, por si só, ser considerada um fator de limitação para a sobrevivência e para a qualidade de vida. As diferenças no nível de alfabetização, entre os sexos, refletem a organização social do começo do século que bloqueou o acesso à escola aos mais pobres e às mulheres. $O$ amplo acesso aos meios de alfabetização, além de uma questão de cidadania, poderia propiciar maior receptividade, por parte destes idosos, aos programas de educação em saúde e também alguma proteção contra as disfunções cognitivas que os afetam com frequência ${ }^{(11-12)}$.

Aliadas à baixa escolaridade, observaramse desvantagens socioeconômicas. Embora reconhecendo a limitação do presente estudo no que se refere à generalização dos resultados, outros estudos apontaram idosos em condições semelhantes. Em um estudo realizado no município de São Paulo, $57 \%$ dos idosos da amostra tinham condições financeiras aproximadas à realidade dos desta pesquisa ${ }^{(13)}$. Em Campinas-SP, cerca de 53\% dos idosos tinham como renda mensal um a três salários mínimos $^{(14)}$.

Observou-se nesta investigação que $82 \%$ dos idosos tinham o Sistema único de Saúde como referência para tratamento de doenças. Com o crescente aumento da população idosa, associado a múltiplas patologias crônicas, acarreta maior utilização do sistema de saúde, configurando-se, portanto, como o grande desafio para o sistema de saúde ${ }^{(15)}$.

A principal causa de mortalidade e morbidade em idosos no Brasil são as doenças crônicas, que normalmente têm desenvolvimento lento, duram períodos extensos e apresentam efeitos de longo prazo, difíceis de se prever. À semelhança dos demais países ricos, as pesquisas sugerem que as condições complexas, como hipertensão, diabetes e depressão, irão impor uma carga ainda maior no futuro ${ }^{(16)}$.

Em nosso estudo, observamos percentual expressivo de idosos com história de hospitalização. Embora em determinadas circunstâncias a hospitalização seja a única 
possibilidade para o tratamento do idoso, ela tem como repercussões a diminuição da capacidade funcional, a recuperação mais lenta e prolongada, a demanda de tecnologias de alto custo aumentando os gastos com assistência médica e a necessidade de recursos humanos capacitados. Destaca-se, ainda, a dificuldade na continuidade da atenção após alta, tendo em vista que a maioria necessitará de alguma forma de assistência para os cuidados básicos do cotidiano na atenção primária ${ }^{(17)}$.

A avaliação da capacidade funcional de pessoas idosas pelos serviços de saúde no Brasil é uma recomendação da Política Nacional de Atenção à Saúde do Idoso ${ }^{(18)}$. Segundo esta, a avaliação deve ser feita de forma integrada e envolvendo diferentes profissionais de saúde. Assim, é relevante que a equipe multiprofissional e interdisciplinar envolvida possa desenvolver ações que promovam o redirecionamento da prática assistencial, na perspectiva de oferecerem um cuidado em saúde voltado à sua realidade e naquilo que os idosos de fato apresentam como necessidades, no intuito de que promovam um cuidado efetivo na aplicação de tecnologias e na promoção da saúde ${ }^{(18)}$.

A avaliação da capacidade funcional é fundamental para determinar o comprometimento e a necessidade de auxílio para as atividades de manutenção e promoção da própria saúde e de gestão do ambiente domiciliar por parte dos idosos, podendo guiar as políticas públicas de atenção à saúde e as políticas sociais para este segmento ${ }^{(19)}$. Observou-se maior prevalência nas AIVD do que nas ABVD, o que está de acordo com outras pesquisas ${ }^{(20)}$. Hierarquicamente, as perdas ocorrem de atividades instrumentais de vida diária para atividades básicas de vida diária devido às AIVD exigirem maior integridade física e cognitiva quando comparadas às $A B V D^{(21-22)}$.

Neste trabalho, a única variável que apresentou efeito estatisticamente significativo na visita domiciliar foi o fato de passarem em consulta médica na Unidade Básica de Saúde, indicando que idosos que passavam em consulta médica na Unidade Básica de Saúde tiveram mais chances de receber visita domiciliar.

Quando indivíduos idosos não conseguem comparecer ao serviço de saúde em razão de alguma incapacidade, a visita domiciliária - atividade externa à Unidade Básica de Saúde (UBS) ${ }^{(22)}$ mais realizada pela equipe de saúde - assume importância crucial, pois permite conhecer in loco a realidade e as necessidades das famílias e de seus membros. Trata-se de uma ferramenta de cuidado e promoção da saúde, de busca ativa e identificação da demanda reprimida, de diagnóstico local e de planejamento de ações a partir da realidade, de mediação entre as famílias e as equipes de saúde ${ }^{(23)}$.

Um estudo realizado com 1.593 idosos no município de Bajé, RG, em 2008, evidenciou que a equipe de Enfermagem foi responsável por $78 \%$ das visitas domiciliares realizadas para prestar cuidados $^{(24)}$. Entre as atividades realizadas pelos enfermeiros está a visita domiciliar que permite conhecer o contexto social e identificar as necessidades de saúde das famílias atendidas por estes profissionais, possibilitando uma maior aproximação com os determinantes do processo saúde-doença. Sendo assim, acredita-se que este seja um espaço promissor para a promoção da saúde ${ }^{(25)}$. A visita domiciliar é "utilizada com o intuito de subsidiar a intervenção no processo de saúde-doença de indivíduos ou no planejamento de ações visando à promoção da saúde da coletividade ${ }^{(25)}$.

\section{CONSIDERAÇÕES FINAIS}

Em síntese, os resultados do presente estudo mostraram que os idosos avaliados eram mulheres na faixa etária de 60 a 69 anos, com baixa escolaridade, renda familiar insuficiente, vivendo sem cônjuge, dependentes do Sistema Único de Saúde, presença de doenças crônicas, com destaque para hipertensão arterial, e incapacidade para desempenho de atividades instrumentais da vida diária. Os idosos que passavam em consulta médica na Unidade Básica de Saúde tendem a receber visita domiciliar da equipe de saúde. A visita domiciliar tem sido um dos instrumentos historicamente mais utilizados no âmbito da enfermagem comunitária e atualmente no quotidiano da Estratégia Saúde da Família. Tal ação coloca a família como o centro do cuidado, assistindo todas as fases do ciclo de vida.

\section{REFERÊNCIAS}

1. Marque GQ, Freitas IBA. Experiência-piloto de assistência domiciliar: idosos acamados de uma Unidade Básica de Saúde, Porto Alegre, Brasil. Rev Esc Enferm USP. 2009;43(4):825-

32. https://doi.org/10.1590/5008062342009000400013

2. Coelho JMF. Saúde do idoso. In: Rouquayrol MZ, Gurgel M. Epidemiologia e saúde. Rio de Janeiro: Medbook; 2014. p. 401-21. 
3. Silveira TM, Caldas CP, Carneiro TF. Cuidando de idosos altamente dependentes na comunidade: um estudo sobre cuidadores familiares principais. Cad Saúde Pública. 2006; 22(8):1629-38.

https://doi.org/10.1590/S0102311X2006000800011

4. Carneiro LAF, Campino ACC, Leite F, Rodruges CG, Santos GMM, Silva ARA. Envelhecimento populacional e os desafios para o sistema de saúde brasileira. São Paulo: Instituto de Estudos de Saúde Suplementar; 2013 [citado em 10 dez 2015]. Disponível em: www.iess.org.br/envelhecimentopop2013.pd $\underline{f}$

5. Rehem TCMSB, Trad LAB. Assistência domiciliar em saúde: subsídios para um projeto de atenção básica brasileira. Cienc Saúde Coletiva. 2005;10 Supl:231-42. https://doi.org/10.1590/S1413$\underline{81232005000500024}$

6. Silva KL, Sena R, Leite JCA, Seixas CT, Gonçalves AM. Internação domiciliar no Sistema Único de Saúde. Rev Saúde Pública. 2005;39(3):391-7. https://doi.org/10.1590/S0034$\underline{89102005000300009}$

7. Oliveira MAC, Pereira IC. Atributos essenciais da Atenção Primária e a Estratégia Saúde da Família. Rev Bras Enferm. 2013;66(esp):15864. https://doi.org/10.1590/S0034$\underline{71672013000700020}$

8. Katz S, Ford AB, Moskowitz RW, Jackson BA, Jaffe MW. Studies of illness in the aged. The index of ADL: a standardized measure of biological and psychosocial function. JAMA. 1963;185(12):914-9. https://doi.org/10.1001/jama.1963.03060120 $\underline{024016}$

9. Lawton MP, Brody EM. Assessment of older people: self-maintaining and instrumental activities of daily living. Gerontologist. 1969 [citado em 11 dez 2015];9(3):179-86. Disponível em:

http://gerontologist.oxfordjournals.org/conte nt/9/3 Part 1/179.extract

10. Santos GS, Cunha ICKO. Avaliação da qualidade de vida de mulheres idosas na comunidade. Rev Enferm Cent.-Oeste Min. 2014;4(2):1135-45. https://doi.org/10.19175/recom.v0i0.593

11. Santos MIPO, Griep RH. Capacidade funcional de idosos atendido em um programa do SUS em Belém (PA). Cienc Saúde Coletiva.
2013;18(3):753-761.

https://doi.org/10.1590/S1413-

81232013000300021

12. Santos GS, Cunha ICKO. Capacidade funcional de idosos em Unidade Básica de Saúde do município de São Paulo. REAS. 2013 [citado em 16 dez 2015];2(3):67-76. Disponível em: http://www.uftm.edu.br/revistaeletronica/in dex.php/enfer/article/view/590

13. Minosso JSM, Amêndola F, Alvarenga MRM, Oliveira MAC. Prevalência de incapacidade funcional e independência em idosos em um centro de saúde-escola da Universidade de São Paulo. Cogitare Enferm. 2010;15(1)12-8. https://doi.org/10.5380/ce.v15i1.17138

14. Zaitune MPA, Barros MBA, César CLG, Carndina L, Goldbaum M. Hipertensão arterial em idosos: prevalência, fatores associados e práticas de controle no Município de Campinas, São Paulo, Brasil. Cad Saúde Pública2006; 22(2):285-294. https://doi.org/10.1590/s0102311X2006000200006

15. Veras RP. Envelhecimento populacional contemporâneo: demandas, desafios e inovações. Rev Saúde Pública. 2009;43(3):548-54. https://doi.org/10.1590/S003489102009005000025

16. Veras RP. Estratégias para o enfrentamento das doenças crônicas: um modelo em que todos ganham. Rev Bras Geriatr Gerontol. 2011;14(4)779-786. https://doi.org/10.1590/S1809$\underline{98232011000400017}$

17. Pagotto V, Silveira EA, Velasco WD. Perfil das hospitalizações e fatores associados em idosos usuários do SUS. Cienc Saúde Coletiva. 2013;18(10):3061-3070.

https://doi.org/10.1590/S141381232013001000031

18. Brasil. Lei № 10.741 , de $1^{\circ}$ de outubro de 2003. Dispõe sobre o Estatuto do Idoso e dá outras providências. Diário Oficial União. 3 out 2003 [citado em 3 nov 2015] Disponível em: http://www2.camara.gov.pdf

19. Barbosa BR, Almeida JM, Barbosa MR, RossiBarbosa LAR. Avaliação da capacidade funcional dos idosos e fatores associados à incapacidade. Cienc Saúde Coletiva. 2014;19(8):3317-3325. https://doi.org/10.1590/141381232014198.06322013 
20. Dellaroza MSG, Pimenta CAM, Duarte YA, Lebrao ML. Dor crônica em idosos residentes em São Paulo, Brasil: prevalência, características e associação com capacidade funcional e mobilidade (Estudo SABE). Cad Saúde Pública. 2013;29(2):325-334. https://doi.org/10.1590/S0102311X2013000200019

21. Pilger C, Menon UM, Mathias TAF. Capacidade funcional de idosos atendidos em unidades básicas de saúde do SUS. Rev Bras Enferm. 2013;66(6):907-13. https://doi.org/10.1590/S003471672013000600015

22. Santos GS, Cunha ICKO. Capacidade funcional e sua mensuração em idosos: uma revisão integrativa. REFACS. 2014;2(3):219-29. https://doi.org/10.18554/refacs.v2i3.1215

23. Magalhães KA, Giacomin KC, Santos WJ, Firmo JOA. A visita domiciliária do agente comunitário de saúde a famílias com idosos frágeis. Cienc Saúde Coletiva. 2015;20(12):3787-96. https://doi.org/10.5216/ree.v16i1.20260

24. Fernandes MT, Soares SM. $O$

Desenvolvimento de Políticas Públicas de atenção ao idoso no Brasil. Rev Esc Enferm USP. 2012;46(6):1494-502. https://doi.org/10.1590/S008062342012000600029

25. Thumé E, Facchini LA, Tomasi E, Vieira LAS. Assistência domiciliar a idosos: fatores associados, características do acesso e do cuidado. Rev Saúde Pública. 2010;44(6):1102111. https://doi.org/10.1590/S0034$\underline{89102010005000038}$

Nota: O presente artigo é parte integrante da Tese de Doutorado intitulada 'Atendimento ao Idoso na Atenção Básica e as Competências do Enfermeiro'. Defendida em 28/08/2014 na Escola Paulista de Enfermagem da Universidade Federal de São Paulo (UNIFESP).

Recebido: 22/01/2016

Versão final: $14 / 08 / 2017$

Aprovado em: 14/08/2017

Endereço para Correspondência:

Rua Tuitui no 928 Apto 153 - Tatuapé

CEP: 03081-015 São Paulo/SP - Brasil

E-mail: enf.gerson@hotmail.com 